\title{
The role of immunotherapy in combination with oligometastasis-directed therapy: a narrative review
}

\author{
William Tyler Turchan^, Steven J. Chmura \\ Department of Radiation and Cellular Oncology, University of Chicago, Chicago, IL, USA \\ Contributions: (I) Conception and design: SJ Chmura; (II) Administrative support: SJ Chmura; (III) Provision of study materials or patients: None; (IV) \\ Collection and assembly of data: WT Turchan; (V) Data analysis and interpretation: All authors; (VI) Manuscript writing: All authors; (VII) Final \\ approval of manuscript: All authors. \\ Correspondence to: Steven J. Chmura, MD, PhD. 5758 S Maryland Ave, Chicago, IL 60637, USA. Email: schmura@radonc.uchicago.edu.
}

\begin{abstract}
Metastatic disease is a significant cause of morbidity and mortality among patients with cancer. Patients with oligometastatic cancer represent a subset of the metastatic population with a limited amount of disease that has metastasized distantly and progresses at a slow pace and thus has the potential to be cured with metastasis-directed local therapy. Recent studies examining the role of metastasis-directed therapy in patients with oligometastatic disease have primarily focused upon treatment with ablative doses of radiation, commonly referred to as stereotactic body radiation therapy (SBRT). While the use of SBRT to treat oligometastases has increased considerably in recent years, the benefit of this approach has yet to be confirmed in phase III randomized controlled trials; moreover, distant failure remains a significant problem in patients with oligometastatic disease treated with SBRT. Given the propensity for distant failure in patients with oligometastatic disease treated with SBRT, there is growing interest in the utility of combining SBRT with systemic agents such as immunotherapy. Immunotherapy, and specifically immune checkpoint blockade (ICB), represents a rapidly evolving systemic therapy option with a growing number of indications among patients with metastatic disease; however, despite its promise, only a minority of patients respond to ICB and among those who do, the majority eventually progress. SBRT and ICB are both dependent upon, and have the ability to shift, the balance between antitumor immune surveillance and immunosuppressive states in the tumor and tumor microenvironment. As a result, it has been speculated that SBRT and ICB have the potential to act synergistically when used in combination. SBRT has been demonstrated to be safe in combination with ICB in studies with short-term follow-up and although additional research is needed, preliminary prospective data support the potential efficacy of this approach. In addition to confirming the safety and efficacy of SBRT in combination with immunotherapy, further studies are needed to determine how to maximize the therapeutic ratio of this treatment paradigm for the full potential of immunotherapy in the oligometastatic population to be realized.
\end{abstract}

Keywords: Immunotherapy; oligometastatic; radiation therapy; stereotactic body radiation therapy (SBRT); stereotactic ablative radiation therapy (SABR)

Submitted Aug 05, 2020. Accepted for publication Nov 19, 2020.

doi: $10.21037 /$ apm-20-1528

View this article at: http://dx.doi.org/10.21037/apm-20-1528

$\wedge$ ORCID: 0000-0001-9537-6980. 


\section{Appeal of immunotherapy for treatment of patients with oligometastatic disease}

\section{Oligometastatic disease}

Among patients afflicted with cancer, metastatic disease is a significant cause of morbidity and mortality; it has been estimated that metastases account for up to $90 \%$ of cancer-related mortality (1). Historically, it was believed that metastatic disease is characterized by a predictable pattern of progression that portends a consistently poor prognosis for patients, though an increasingly nuanced understanding of the biology of cancer metastasis has challenged this myopic view. Long before the development of contemporary models of cancer metastasis, Halsted proposed that metastatic progression occurs in an orderly fashion in which primary tumor cells first spread regionally to draining lymph nodes, where tumor cells accumulate until capacity in the lymph nodes is reached and tumor cells spill out to the next region of drainage (2). This idea was directly challenged by the theory that cancer metastasis does not occur in an orderly fashion and that distant spread occurs early in its natural history, which was most famously articulated by Fisher (3). These theories were subsequently reconciled by Hellman who proposed the spectrum hypothesis, which postulated the existence of a continuum of metastatic progression ranging from disease with a tendency to remain local to that with a propensity for early distant metastasis (4). Shortly thereafter, this hypothesis was refined by Hellman and Weichselbaum who proposed the existence of an oligometastatic state in which patients present with a limited amount of disease that has metastasized distantly, but is unlikely to progress rapidly (5).

While it is not well-characterized in the historical literature, recent data suggest that presentation with oligometastatic disease is not uncommon. A review of several first-line systemic therapy trials for patients with metastatic breast cancer found that approximately $50 \%$ of enrolled patients initially had two or fewer metastatic sites and up to $75 \%$ had four or fewer (6). Similar findings have been reported in several other common histologies including non-small cell lung cancer (NSCLC) (7), prostate cancer (8), and renal cell carcinoma (9) suggesting that the oligometastatic state may be relatively common. Given its seeming ubiquity across multiple histologies, the oligometastatic state represents an area that is both amenable to further research and where the results of such inquiry have the potential to significantly improve outcomes for a large number of patients. We present the following article in accordance with the NARRATIVE REVIEW reporting checklist (available at http://dx.doi.org/10.21037/ apm-20-1528).

\section{Metastasis-directed therapy for oligometastatic disease}

As a result of the hypothesized frequency of the oligometastatic state, there has been significant interest in the optimal management of patients who fall into this category. Given the inherently limited burden of disease in patients with oligometastases, local therapy has been attempted as a therapeutic option dating back to a pulmonary metastasectomy following the resection of a chest wall sarcoma, as described by Weinlechner et al. in 1882 (10). Since that time, surgery has been utilized extensively in patients with limited metastatic disease with curative intent, primarily among patients with pulmonary, liver, and adrenal metastases and less commonly among patients with other sites of extracranial metastasis. In fact, the most robust long-term survival data available among patients with limited metastatic disease come from patients with colorectal cancer who have undergone metastasectomy of liver metastases (11). Moreover, multiple other reported experiences have demonstrated the potential for significant rates of long-term ( $>10$ years) overall survival (OS) with complete resection of metastatic disease (12-14).

While metastasectomy does have a history of success in a select population of patients with low burden metastatic disease, many patients with metastatic cancer are not able to undergo metastasectomy as a result of their medical comorbidities and/or disease that is not amenable to resection. As previously noted, while frequently used for pulmonary, liver, and adrenal metastases, metastasectomy has not typically been utilized in patients with other sites of extracranial metastasis; moreover, metastasectomy has typically been reserved for patients with one site of metastatic disease rather than several. Thus, the search for other methods of effective local therapy in patients who are not candidates for metastasectomy drove the development of hypofractionated courses of precisely-delivered, ablative doses of radiation therapy, often referred to as stereotactic body radiation therapy (SBRT) or stereotactic ablative radiation therapy (SABR). In recent years, SBRT/SABR (hereinafter referred to simply as "SBRT") has become a popular and increasingly utilized option for metastasisdirected therapy (15). Although several prospective series with long-term follow-up have examined the role of SBRT in patients with oligometastatic disease (16-20), the 
randomized data supporting the role of metastasis-directed SBRT remain scarce. SABR-COMET, a phase IIR screening trial, which randomized 99 patients with a controlled primary tumor and 5 or fewer distant metastases to standard of care (SOC) therapy with or without SBRT in a 2:1 ratio demonstrated significant improvements in median progression-free survival (PFS) and OS with the addition of metastasis-directed SBRT to SOC therapy $(21,22)$. While the results of SABR-COMET are undoubtedly exciting, several caveats should be considered in its interpretation, including the variety of histologies treated and imbalances between the SBRT and SOC arms $(21,22)$. Moreover, it is of paramount importance to be mindful of its intent as a phase II screening trial, to identify potentially significant outcomes for further testing in confirmatory phase III trials (23).

Although the results of SABR-COMET are encouraging and strengthen the conclusions of smaller, disease-specific, phase II studies that demonstrate PFS, and in some cases OS, benefits among patients treated with SBRT to low volume metastatic disease (24-27) further validation of these results in the form of disease-specific, phase III randomized trials is needed. Moreover, given the relatively limited number of histologies represented in the current phase II studies along with the fact that the vast majority of patients represented in the currently reported experiences had a low burden of disease relative to upper limit of 5 metastases commonly used to define the oligometastatic state (28) further work is needed to establish optimal management for many patients with limited burden metastatic disease. Finally, given the propensity for long-term distant failure despite local therapy, additional strategies are needed in order to attain durable disease control in patients with oligometastatic disease. One such strategy is the combination of SBRT with systemic agents such as immunotherapy.

\section{The rapidly evolving landscape of immunotherapy}

Immunotherapy has been utilized to treat patients with cancer for several decades. Its indications and utilization have expanded massively over the last few years with the largest gains achieved in the use of immune checkpoint blockade (ICB) to block cytotoxic T-lymphocyte antigen-4 (CTLA-4) and programmed cell death protein-1 (PD-1) along with its ligand (PD-L1). Since ipilimumab, a monoclonal antibody that targets CTLA-4, was first approved for use in patients with advanced melanoma in 2011 (29) the proportion of patients with advanced or metastatic disease eligible for treatment with ICB has increased from approximately $1.5 \%$ to greater than $40 \%$, as of 2018 (30). Moreover, immunotherapy has been demonstrated to improve outcomes and in some instances produce long-term disease-free intervals, not only in melanoma, but in a variety of different advanced malignancies (30-38). Though the data supporting the benefit of ICB in patients with metastatic disease have increased rapidly, the role of ICB remains uncertain in many histologies. Moreover, despite its triumphs, only approximately $20-25 \%$ of patients with metastatic disease respond to ICB $(32,39-42)$ and among those who do, the majority eventually progress (43-46), underscoring the need to continue to improve upon the advances that the have been made in the use of immunotherapy to treat patients with metastatic disease. One such strategy is the liaison of immunotherapy with local therapy in order to improve response rates.

Perhaps one of the largest advances in the use of ICB in the treatment of cancer patients has come in the form of durvalumab as a consolidative therapy following definitive chemoradiation for patients with non-metastatic, unresectable, NSCLC. In this setting ICB has been shown to provide a significant PFS and OS benefit $(47,48)$. Given its demonstrated efficacy in the eradication of microscopic disease following chemoradiation in the locally advanced setting, the combination of ICB with radiation therapy represents a logical strategy to maximize therapeutic response in patients with low-volume metastatic disease. The potential benefit of this combination is further supported by the fact that immunotherapy has been demonstrated to be most efficacious in the setting of lowvolume disease (49), while ablative doses of radiation are capable of significant cytoreduction $(50,51)$. Moreover, the abscopal effect, which describes the response of tumors outside of the irradiated field following radiation therapy, further bolsters the rationale for the union of immunotherapy and radiation therapy.

First described in 1953 by Mole et al. (52) the abscopal effect remained somewhat of an enigma for decades, rarely and unpredictably occurring in patients treated with radiation therapy (53). One hypothesis attempting to explain the abscopal effect postulated that it was, at least in part, a T-cell-dependent, immune-mediated event (54). Accordingly, abscopal effects were described by Postow et al. in 2012 in a patient with metastatic melanoma with progression of disease on maintenance ipilimumab who was 
treated with 28.5 Gy over 3 fractions to a painful paraspinal metastasis. Following radiation therapy, the patient was noted to have regression of not only her treated paraspinal lesion, but also other metastatic lesions that were not irradiated (55). This and other reports of robust abscopal effects following the combination of ICB with SBRT has invigorated interest in this liaison as a potential means to induce widespread response to SBRT in patients with oligometastatic disease.

\section{Methods}

The primary objective of this narrative review was to characterize the current role of SBRT in combination with immunotherapy among patients with oligometastatic disease as well as potential future directions in this area. Specifically, we aimed to review the data supporting the biologic basis for combining SBRT with immunotherapy. We subsequently assessed the current data to support the safety and efficacy of this approach. Finally, we reviewed emerging data to support potential future methods to improve the therapeutic ratio of this liaison. Using $\mathrm{MeSH}$ terms and keywords including, but not limited to: immunotherapy, ICB, oligometastatic, radiation therapy, SBRT, and SABR, we constructed search terms, which were used to query PubMed as well as Google Scholar. Data in the narrative portion of the review included English-language publications between 1999 and 2020. ClinicalTrials.gov, which is an online registry of clinical trials run by the United States National Library of Medicine at the National Institute of Health, was also used to identify relevant ongoing prospective studies.

\section{Biologic basis for the combination of immunotherapy with oligometastasis-directed therapy}

\section{Role of T-cells in antitumor response}

In order to improve the efficacy of immunotherapy in combination with oligometastasis-directed therapy, an understanding of the underlying biologic effects of these therapies is required. The role of the immune system in response to treatment is well established; immuneexcluded tumors, which are characterized by lack of T-cell infiltration, including low levels of helper (TH1) and cytotoxic (CD8+) T-cells, have been shown to be associated with decreased therapeutic response (56). Using patient- derived xenografts, Lee et al., demonstrated the importance of cytotoxic T-cells for tumors to respond to ablative doses of radiation. In their experiments, Lee et al. demonstrated that immunocompetent murine xenografts treated with ablative doses of radiation responded to treatment, while nude mice and cytotoxic T-cell-depleted wild-type mice did not (57). Others have similarly shown the importance of T-cell infiltration $(58,59)$ for response to radiation therapy and accordingly, levels of tumor infiltrating lymphocytes (TILs) have been demonstrated to be prognostic in patients treated with radiation across multiple histologies (60-63). Unsurprisingly, T-cell infiltration has also been demonstrated to play a significant role determining response to ICB, as heavily T-cell infiltrated or "hot" tumors have been demonstrated to have a higher rate of response to ICB compared to less T-cell infiltrated or "cold" tumors (64). Thus, T-cells play an important role in antitumor surveillance and degree of T-cell infiltration is a significant determinant of therapeutic response to both radiation therapy and immunotherapy.

\section{Mechanisms of ICB}

Within the tumor microenvironment, T-cell activation is regulated by tumor cells, macrophages, and antigen presenting cells (APCs), such as dendritic cells (DCs), all of which have the ability to form ligand-receptor interactions with T-cells resulting in either immunestimulation or immunosuppression. These ligand-receptor interactions are known as immune checkpoints and blockade of these checkpoints (ICB) has revolutionized the role of immunotherapy in the treatment of cancer. Currently approved ICB agents have one of two targets, CTLA-4 or PD-1/PD-L1 (65). CTLA-4 is expressed constitutively on immunosuppressive, regulatory T-cells (Tregs) and is upregulated in activated T-cells. CTLA4 binds the same ligands as the co-stimulatory T-cell receptor $\mathrm{CD} 28$; however, with greater affinity allowing it to outcompete CD28. Following activation, T-cells upregulate CTLA-4 resulting in an increase in inhibitory signals and a decrease in stimulatory signals, ultimately decreasing immune surveillance (66). As such, CTLA-4 blockade with antagonistic antibodies can decrease immunosuppression and increase antitumor immune surveillance. PD-1, which is also expressed on T-cells, transmits pro-apoptotic signals in activated T-cells and anti-apoptotic signals in Tregs in response to its ligand PD-L1. PD-L1 is frequently upregulated in cancer cells as a mechanism of immune 
evasion (66). As such, antibodies targeting PD-1/PDL1 are another frequently used form of ICB. Overall, the efficacy of ICB relies upon several relationships within the tumor microenvironment. Similarly, multiple important interactions within the tumor microenvironment underlie tumor response to radiation therapy.

\section{Immune response to ionizing radiation}

Following treatment with ionizing radiation, a complex cascade of events occurs within tumors and the surrounding tumor microenvironment with the potential to either increase or suppress antitumor immune surveillance. Radiation and resulting cell death results in cytosolic release of DNA, which is recognized by cyclic GMPAMP synthase (cGAS), resulting in type I interferon (IFN) production via the stimulator of interferon genes (STING) pathway (67). In addition to toll-like receptor-dependent stimulation, STING promotes IFN production, which has a number potentially immunostimulatory actions, including the recruitment of CD8+ T-cells and activation of DCs, which prime CD8+ T-cells for antitumor response (68). However, prolonged IFN exposure may stimulate tumor survival factors (69) and can also upregulate tumor PDL1 expression resulting in immunosuppression (70,71). In fact, increased expression of PD-L1 following irradiation has previously been recognized as a significant cause of resistance to radiation therapy that can potentially be overcome with PD-L1 blockade (72). Consequently, radiation therapy-induced activation of the STING pathway has multiple effects, some of which bolster antitumor response, while others, such as upregulation of PD-L1 contribute to immune evasion and could potentially be targeted by ICB to increase therapeutic response.

Ionizing radiation also results in the secretion of numerous cytokines and chemokines into the tumor microenvironment, which similarly can have conflicting effects on antitumor immune surveillance. Factors secreted into the tumor microenvironment following radiation recruit a host of immune-related cells to the tumor microenvironment including DCs, macrophages, and CD8+ T-cells, all of which have the potential to enhance antitumor response $(73,74)$. Given its ability to promote the migration of effector T cells, SBRT also has the ability increase tumor T-cell infiltration, turning "cold" tumors "hot" (73-78), without eliminating resident T-cells in inflamed tumors (79). In addition, ionizing radiation upregulates cellular adhesion molecules in endothelial cells, which can further increase
T-cell infiltration $(73,76)$. Conversely, factors secreted into the tumor microenvironment following radiation also have the potential to result in the efflux of potentially immunostimulatory cell populations and attract Tregs and myeloid-derived suppressor cells (MDSCs) (54,78,80-84). Tregs secrete TGF- $\beta$ and IL-10, have the ability to suppress effector T-cell function $(85,86)$, and have been demonstrated to be associated with suppressed antitumor immune response and poor clinical outcomes $(87,88)$. Moreover, Tregs enhance the function of MDSCs, which have been demonstrated to have important roles in promoting tumor invasion and metastasis (89) and also induce Tregs (90). Tregs notably constitutively express CTLA-4 and selective depletion of Tregs in the tumor microenvironment has been demonstrated in response to ipilimumab (91). Thus, given the prominent role that Tregs play in inhibiting antitumor surveillance following radiation therapy, ICB-mediated depletion of Tregs represents an additional opportunity to improve therapeutic response to radiation therapy.

Another important component of the tumor microenvironment, tumor-associated macrophages (TAMs), are a highly plastic class of cells that have been implicated in tumor immune surveillance. Historically, TAMs have been described as polarized into either M1 or M2 subtypes, with M1 macrophages acting in a proinflammatory, antitumor fashion, and M2 macrophages acting in an immunosuppressive fashion (92). While it has recently been suggested that this binary classification is not entirely accurate and that a spectrum of activation of TAMs exists (93), high doses of radiation have been demonstrated to induce an M2-like phenotype $(94,95)$ and depletion of TAMs prior to radiation has been demonstrated to increase antitumor activity (96), while M1-like macrophages promote recruitment and activation of CD8+ T-cells following radiation (76). As such, there is interest in the combination of radiation therapy with, cabiralizumab a monoclonal antibody antagonist of colony-stimulating factor 1 receptor (CSF-1R), which has been demonstrated to skew TAM populations towards an M2-like phenotype (97). In summary, ionizing radiation results in several changes in the tumor microenvironment, some of which promote antitumor immune surveillance, while others inhibit antitumor response; in many cases these effects are targetable via immunotherapy. Thus, the utilization of immunotherapy to skew the tumor microenvironment towards an immunostimulatory, antitumor phenotype and away from an immunosuppressive protumor phenotype 
represents an enticing strategy to improve therapeutic response to radiation therapy.

In addition to its aforementioned effects on the tumor microenvironment, radiation releases tumor antigens as well as damage-associated molecular patterns (DAMPs) which are well-established to have an important role in improved DC function and T-cell priming $(98,99)$. Moreover, cytoreduction following SBRT represents another potentially important means by which radiation can increase immune surveillance and improve outcomes with ICB. Given that TILs have been demonstrated to be relatively radioresistant (79), SBRT provides a potential mechanism for the selective elimination of tumor cells. SBRT has been show to decrease the ratio of tumor cells to non-exhausted effector T-cells, which has been demonstrated to correlate directly with PFS (100). Moreover, given that increasing antigen burden has been demonstrated to promote T-cell exhaustion, cytoreduction provides a possible means by which to lower antigen burden and potentially increase T-cell re-invigoration (50,51). Given that immunotherapy has been demonstrated to be most efficacious in the setting of low-volume disease (49), the cytoreductive properties of SBRT provide an important mechanism by which SBRT and immunotherapy may act synergistically.

\section{Prospective data evaluating combination of immunotherapy with oligometastasis-directed therapy}

\section{Safety of ICB with SBRT}

Given the relatively novel concept of combining ICB with SBRT in patients with metastatic disease, the safety of combination therapy is of significant concern, especially given the uncertain clinical benefit of this regimen. Notably, SABR-COMET reported significantly more treatmentrelated grade 2 or higher adverse events among patients treated with SBRT (29\%) compared to those treated with SOC therapy only (9\%). Moreover, 3 patients (5\%) treated with SBRT experienced grade 5 treatment-related adverse events (one patient with radiation pneumonitis, one patient with a post-treatment pulmonary abscess, and one patient with a subdural hemorrhage after surgical repair of a SBRT-related perforated gastric ulcer), while no patients in the SOC arm experienced treatment-related death (21). Additionally, immunotherapy is associated with its own set of potential complications, with the most common immune-related adverse events (irAEs) being dermatitis
(101) and colitis (102) followed by endocrinopathy (103) and hepatotoxicity (104). A less common, but potentially serious irAE is pneumonitis. Pneumonitis related to ICB is well-documented and has the potential to cause significant morbidity and in some instances mortality (105109). Moreover, given that pneumonitis is a potentially fatal complication of metastasis-directed SBRT (21) and radiation recall pneumonitis has been reported in patients who receive ICB well after completion of radiation therapy (110) the potential for serious toxicity related to the combination of ICB with SBRT exists driving the need for clinical trials.

Multiple prospective studies aimed at evaluating the safety of ICB in combination with SBRT have been performed. Our institution performed a phase I study evaluating the combination of pembrolizumab with SBRT in patients with advanced solid tumors that had progressed on SOC therapy. In this study, 73 of 79 enrolled patients received SBRT and at least one cycle of pembrolizumab, which began within 7 days of completion of SBRT. A total of 2-4 metastases were treated per patient with each metastasis receiving 30-50 Gy in 3-5 fractions (identical to the doses and normal tissue constraints employed with NRG BR001), with dose and fractionation dependent upon metastasis location. Notably, as opposed to other trials evaluating the role of SBRT, metastases greater than 65 $\mathrm{mL}$ were partially-irradiated with only $65 \mathrm{~mL}$ of any tumor receiving the prescription dose in an attempt to minimize toxicity. With a median follow up of 5.5 months, doselimiting toxicity (DLT) was seen in only 6 patients $(9.7 \%$; $\mathrm{n}=3$ grade 3 pneumonitis, $\mathrm{n}=2$ grade 3 colitis, and $\mathrm{n}=1$ grade 3 hepatic toxicity), with no dose reductions required (111). The overall objective response rate (ORR) of non-irradiated metastases was $13.2 \%$. With additional follow-up, it was determined that the degree of irradiated tumor response was correlated with outcome, with median overall survivals of $17.8,9.1$, and 3.4 months among patients with complete/ partial local response, stable local disease, and progressive local disease, respectively (112). Thus, these results suggest not only that metastasis-directed SBRT was well-tolerated, but also that degree of local response to SBRT is prognostic of survival.

Additional smaller, non-randomized, prospective studies evaluating the safety of ICB in combination with SBRT have been reported with similar results. For instance, a phase I study performed at the University of Pennsylvania enrolled 22 patients with metastatic melanoma who received SBRT (6-8 Gy per fraction for 2-3 fractions, 
Table 1 Comparative studies of ICB with SBRT in patients with oligometastatic disease

\begin{tabular}{|c|c|c|c|c|}
\hline Study/sponsor & Design & Histology (enrollment) & ORR & Toxicity \\
\hline Regeneron (NCT02383212) & $\begin{array}{l}\text { Phase 1, } 2 \text { ECs; } \\
\text { cemiplimab } \pm \text { SBRT }\end{array}$ & $\operatorname{NSCLC}(n=33, n=20)$ & $40 \%$ vs. $18.2 \%$ (NS) & $\begin{array}{l}\text { NS between groups; one } \\
\text { patient with G5 } \\
\text { pneumonitis }\end{array}$ \\
\hline
\end{tabular}

ICB, immune checkpoint blockade; SBRT, stereotactic body radiation therapy; RCT, randomized controlled trial; ECs, expansion cohorts; NSCLC, non-small-cell lung cancer; HNSCC, head and neck squamous cell carcinoma; ORR, overall response rate; NS, non-significant; G3, grade 3 adverse event; G4, grade 4 adverse event; G5, grade 5 adverse event

dependent upon site of metastasis) in combination with ipilimumab with no DLTs and a partial response, as best response, in $18 \%$ by RECIST (75). An additional study performed at MD Anderson Cancer Center (MDACC) reported only 2 DLTs ( $\mathrm{n}=1$ grade 3 pancreatitis and lipase elevation and $n=1$ grade 3 increase in bilirubin and aspartate aminotransferase) and an ORR of $23 \%$ in patients with advanced solid malignancies treated with 50-60 Gy in 4-10 fractions followed by ipilimumab (113). On the whole, the results of these and other, phase I/II, prospective, nonrandomized studies demonstrate that the combination of ICB with SBRT is seemingly safe with encouraging rates of response (114-118). Moving forward, the results of additional studies investigating the role of SBRT in combination with ICB will be important to further establish the safety of this approach. Moreover, the results of ongoing phase III studies investigating this combination in the definitive setting will likely be informative. Currently, PACIFIC-4 (NCT03833154) and SWOG/NRG S1914 (NCT04214262), both of which randomize patients with early-stage NSCLC with high-risk features to treatment with SBRT with or without consolidative ICB, are currently enrolling $(119,120)$. Notably, patients treated on the experimental arms of these studies receive radiation therapy with biologically effective doses (BEDs) in excess of $100 \mathrm{~Gy}$ in combination with ICB and as a result, the outcomes of these trials will likely provide additional valuable information on the safety of combining ablative doses of radiation with ICB.

\section{Comparative studies evaluating the addition of SBRT to $I C B$}

Although most of the evidence evaluating the role of ICB in combination with SBRT is limited to retrospective series and small, single-arm prospective studies, randomized data are beginning to emerge in this area. The currently reported comparative studies of $\mathrm{ICB} \pm \mathrm{SBRT}$ in patients with oligometastatic disease are shown in Table 1. The first of these to be published, was the PEMBRO-RT trial reported by Theelen et al. in 2019. This multicenter study included 76 patients with metastatic NSCLC randomized to receive pembrolizumab \pm SBRT. Patients in the SBRT arm were treated to a single metastatic site, every other day, with 24 Gy in 3 fractions; pembrolizumab began 7 days after the completion of SBRT. Despite a doubling of ORR at 12 weeks, improvement in 12-week ORR did not reach the prespecified benchmark for meaningful clinical benefit and as a result the trial was negative (36\% vs. $18 \% 12$-week ORR in the experimental vs. control arm, $\mathrm{P}=0.07$ ) (121). The trial also reported median a PFS of 6.6 months in the experimental arm vs. 1.9 months in the control arm $(\mathrm{P}=0.19)$ and median OS of 15.9 months in the experimental arm vs. 7.1 months in the control arm $(\mathrm{P}=0.16)$. The authors did note that upon subgroup analysis by PD-L1 status, the addition of SBRT seemed to drive benefits in median PFS $(\mathrm{P}=0.03)$ and median $\mathrm{OS}(\mathrm{P}=0.05)$ in the $\mathrm{PD}-\mathrm{L} 1$ negative subgroup (121). Perhaps more importantly, no increase in adverse events was observed in the experimental arm. Overall, though a negative trial, PEMBRO-RT supports the 
safety of SBRT in combination with ICB and provides some insight regarding directions for future trials investigating the clinical utility of this combined-modality therapeutic approach.

Similarly, a phase I/II randomized controlled trial of pembrolizumab \pm radiation therapy in patients with metastatic NSCLC was performed at MDACC with results recently reported by Welsh et al. This study randomized 20 patients in the phase I portion and subsequently 80 patients in the phase II portion. Unlike the PEMBRORT trial, pembrolizumab was given concurrently in combined-modality arm. Moreover, patients randomized to receive radiation therapy in this trial were treated with 50 Gy in 4 fractions if deemed feasible, which constitutes a significantly higher BED than the 24 Gy in 3 fractions used in the PEMBRO-RT trial. (However, it should be noted that approximately half of the patients randomized to radiation therapy were deemed to have metastatic lesions that were not amenable SBRT and as a result were treated with a moderately hypofractionated course of 45 Gy in 15 fractions.) Overall, the ORR in the combined-modality arm was not significantly different than that of patients treated with pembrolizumab alone ( $22 \%$ vs. $25 \%, \mathrm{P}=0.99)$, nor was median PFS significantly different (9.1 vs. 5.1 months, $\mathrm{P}=0.52$ ) (122). Like PEMBRO-RT, the authors noted that upon subgroup analysis by PD-L1 status, the addition of radiation therapy seemed to result in a benefit in median PFS (20.8 vs. 4.6 months, $\mathrm{P}=0.004$ ) in the low PDL1 subgroup. Notably, rates of grade 3 or higher toxicity were $30 \%$ and $11 \%$ in the combined-modality arms of the phase I and II portions of the trial, respectively. Moreover, two grade 4 cardiac adverse events (myocardial infarction and subsequent ventricular tachycardia) were noted in one patient treated with combined-modality therapy in the phase II portion of the trial (122). While like PEMBRO-RT, the MDACC trial was negative, this study did importantly add to the data supporting the safety of combined-modality treatment with pembrolizumab. Moreover, it is of note that a recently published pooled analysis of these two randomized trials did demonstrate improved PFS (4.4 vs. 9.0 months, $\mathrm{P}=0.045)$ and $\mathrm{OS}(8.7$ vs. 19.2 months, $\mathrm{P}=0.0004)$ and no new safety concerns with the addition of radiation therapy to pembrolizumab (123). Although these findings are hypothesis-generating, they support the need for future, phase III randomized studies evaluating the role of radiation therapy in combination with ICB.

In another trial of ICB \pm SBRT, 62 patients with metastatic head and neck squamous cell carcinoma were randomized to receive nivolumab \pm SBRT (27 Gy in 3 fractions, delivered every other day to one metastatic lesion). As reported in the aforementioned series, the addition of ICB to SBRT was safe, with no increase in grade 3 or higher toxicity in the experimental arm (13.3\% vs. $9.7 \%$, $\mathrm{P}=0.70)$; however, SBRT did not improve ORR in this study (34.5\% vs. 29.0\%, $\mathrm{P}=0.86$ ), nor were median PFS (1.9 vs. 2.6 months, $\mathrm{P}=0.79$ ) or $\mathrm{OS}(14.2$ vs. 13.9 months, $\mathrm{P}=0.75$ ) improved with the addition of SBRT (124). Moreno et al. also reported the results of a comparative study of patients with metastatic NSCLC treated with cemiplimab \pm SBRT at the $19^{\text {th }}$ World Conference on Lung Cancer in 2018. This study was not randomized, but rather was comprised of a comparison of two separate phase I expansion cohorts in which patients either received cemiplimab alone $(n=20)$ or cemiplimab in combination with SBRT (27 Gy in 3 fractions; $n=33$ ). As was the case in the aforementioned studies, the addition of SBRT to ICB did not appear to result in a significantly higher rate of toxicity, though it is important to note that one patient in the experimental arm experienced grade 5 pneumonitis, which was attributed to treatment; furthermore, the addition of SBRT to ICB once again failed to improve ORR (125).

Overall, the results of the currently reported comparative studies investigating the addition of SBRT to ICB support the safety of combined-modality therapy; however, the data to support the efficacy of this approach remains hypothesisgenerating in nature. It is important to note that the doses of radiation used in these trials (with the exception of the patients in the MDACC trial randomized to combinedmodality therapy in whom 50 Gy in 4 fractions was deemed feasible) resulted in BEDs that are well below that of ablative approaches in which BEDs of $100 \mathrm{~Gy}$ and greater are delivered. Given that ablative BEDs have been demonstrated to be imperative for optimal local control of irradiated metastases (126-129) and that degree of local response to SBRT is an important predictor of survival (112) future randomized studies investigating the role of SBRT in combination with ICB should utilize ablative doses of radiation therapy in an attempt to maximize the potential for therapeutic response with this liaison. Although the currently reported randomized studies do support the safety of SBRT in combination with ICB, it is important that future studies utilizing higher doses of SBRT evaluate this further as these, along with ongoing phase III studies investigating the combination of ablative doses of radiation with ICB in the definitive setting, will help to determine the safety of this approach. However, prospective non- 
randomized data do support the safety of multi-site ablative dose radiation therapy in combination with $\operatorname{ICB}(111,112)$.

\section{Future developments in the role of immunotherapy in the treatment of patients with oligometastatic disease}

\section{Manipulation of the tumor microenvironment}

Although the data reviewed herein support the safety of ICB in combination with SBRT additional data are needed to determine its efficacy. In addition to the need to investigate the use of metastasis-directed ablative doses of radiation therapy in combination with ICB in the prospective, randomized setting, novel strategies are needed to attempt to improve the therapeutic ratio of this combination. One approach to improve outcomes involves utilizing knowledge of the pro-immune surveillance and immunosuppressive actions of SBRT and manipulating the tumor microenvironment such that this balance is favorably shifted.

Given their plastic nature, TAMs represent a component of the tumor microenvironment that have the potential to be targeted in order to shift the balance away from immunosuppression in favor of immune surveillance. Specifically, attempts to polarize TAMs towards an M1like antitumor phenotype and away from an M2-like pro-tumor growth phenotype could potentially enhance the effect of ICB and radiation therapy; granulocytemacrophage colony-stimulating factor (GM-CSF), has been investigated in combination with radiation therapy in this capacity, with initially promising results. In a trial at New York University, patients with metastatic solid tumors were treated with GM-CSF concurrently with radiation therapy (35 Gy in 10 daily fractions) with abscopal responses noted in non-treated metastases in $11(26.8 \%$, 95\% CI: $14.2-42.9 \%)$ of the 41 accrued patients (130). Given that a M2-like phenotype has been demonstrated to be induced in response to high doses of radiation $(94,95)$, attempts to manipulate TAM polarization may be even more effective in combination with SBRT. Moreover, given that increased CD8+ T-cell response and resulting increased IFN production has the potential to upregulate tumor expression of PD-L1 (70), the addition of antiPD-1 therapy to this treatment regimen is logical. As such, our institution, is currently investigating the role of SBRT and nivolumab in combination with cabiralizumab (NCT03431948), a monoclonal antibody antagonist of colony-stimulating factor 1 receptor (CSF-1R), which has been demonstrated to skew TAM populations towards an M2-like phenotype (97). Cabiralizumab has also notably been studied previously in combination with nivolumab in patients with pancreatic cancer (131). Thus, future studies should continue to investigate the polarization of TAMs towards an antitumor phenotype to increase the therapeutic ratio of SBRT in combination with ICB. Another potential opportunity to shift the balance of the tumor microenvironment in favor of antitumor response exists in the form of targeting 4-1BB, a transmembrane glycoprotein on activated effector T-cells, which promotes CD8+ T-cell activity and survival and inhibits Tregs, in response to 4-1BB ligand (4-1BBL, CD137) on APCs. Preclinical data support the potential benefit of targeting 4-1BB; the addition of agonistic 4-1BB monoclonal antibodies to radiation therapy has been demonstrated to significantly increase rates of response in murine breast and lung carcinoma models (132). Moreover, 4-1BB monoclonal antibodies combined with PD-1 blockade and SBRT have been studied in murine melanoma models with encouraging rates of response (133). In addition, impressive responses have been reported clinically following the combination of a 4-1BBL agonist with radiation therapy (134) and PD-1 blockade (135), individually. As result, another strategy to improve the therapeutic response of SBRT in combination with ICB exists in the form of targeting 4-1BB on activated effector T-cells. Accordingly, our institution is currently investigating the addition of a 4-1BBL agonist, urelumab, to nivolumab and SBRT in patients with advanced solid tumors (NCT03431948).

Beyond CSF-1R antagonistic and 4-1BB agonistic approaches, additional methods of targeting the tumor microenvironment in order to maximize antitumor activity are currently under investigation. For instance, TGF- $\beta$ has been demonstrated to induce an immune-excluded phenotype and resulting resistance to PD-L1 (136) and as such, inhibition of TGF- $\beta$ and bispecific antibodies targeting both PD-1 and TGF- $\beta(137,138)$ have been investigated as a potential mechanism of overcoming PD-L1 resistance. Moreover, numerous approaches utilizing PD-1/PD-L1 blockade with other agents targeting effector T-cells and chemokine inhibition have been investigated in addition to a wide variety of combinatory approaches joining PD-1/PDL1 blockade with cytotoxic chemotherapy, small molecule inhibitors, hormone therapy, vaccine therapy, and other ICB agents in order to overcome PD-L1 resistance (139). Overall, strategies to induce a more favorable antitumor, 
pro-immune surveillance tumor microenvironment have the potential to increase the efficacy of combined SBRT and ICB.

\section{Innovations in metastasis-directed SBRT}

In addition to attempts to increase the therapeutic ratio of SBRT and ICB, by altering the tumor microenvironment, improvements in metastasis-directed SBRT have the potential to significantly improve the efficacy of this treatment approach. As previously discussed, given that ablative BEDs are crucial for optimizing the local control of irradiated metastases (126-129) and that local response to SBRT has been correlated with survival (112), randomized studies investigating the role of ablative doses of SBRT in combination with ICB are needed. Historically, technical and practical considerations have largely limited the feasibility of multi-site SBRT using ablative doses for patients with metastatic disease, though several recent innovations have significantly decreased these limitations. First, improvements in image-guided radiation therapy (IGRT) have allowed metastases to be treated with smaller margins (140), increasing the ability to treat multiple metastases in close proximity to high doses safely. Additionally, volumetric modulated arc therapy (VMAT) has been demonstrated to improve treatment conformality, increasing the ability to deliver ablative doses to multiple metastases with sharp dose gradients and highly conformal dose distributions (141). VMAT has also been shown to significantly decrease treatment times, increasing the practicality of treating several metastases in one course of treatment and decreasing the risk of intrafraction motion, which has allowed further reduction of target volume margins (141). On the whole, these technical innovations combined with the realization that partial irradiation of large metastases with ablative doses does not seem to comprise efficacy $(111,112)$ have contributed significantly to the ability to safely deliver ablative-dose SBRT to multiple metastases, which is likely necessary to increase the efficacy of combined SBRT and ICB. Notably, retrospective analysis of patients treated on protocol with multi-site ablative SBRT and ICB using the aforementioned approaches suggests that much higher BEDs, in excess of $360 \mathrm{~Gy}$, can be achieved while respecting normal tissue constraints, and thus, further dose-escalation beyond standard ablative doses may be a feasible method of improving the efficacy of combined SBRT and ICB (142).

In addition to permitting safe dose-escalation of metastasis-directed SBRT, these advancements have also increased the feasibility of simultaneously treating multiple metastases within the same radiation therapy course. Like dose-escalation, the treatment of multiple metastases has the potential to significantly increase response to combined SBRT and ICB. Many of the studies examining the combination of the ICB with SBRT have done so under the premise that radiation can act as an adjuvant that stimulates the activity of ICB, with the goal of achieving abscopal responses at unirradiated sites. However, recent attempts to induce this type of abscopal response with non-ablative doses of radiation in combination with ICB have failed to produce robust therapeutic responses $(121,125,143)$. While, SBRT certainly has the potential for synergy with immunotherapy resulting in effects outside of the irradiated field, the local benefits of ablative radiation suggest that it plays an important role beyond acting as an adjuvant. Given that radiation increases T-cell infiltration of tumors locally (73-78) and is able to re-prime resident T-cells $(73,74,79)$, as well as kill resistant tumor clones (144), targeting all sites of metastasis may be beneficial in patients with oligometastatic disease. Also, given that increasing antigen burden has been demonstrated to promote T-cell exhaustion, maximal cytoreduction through multi-site SBRT has the potential to further lower antigen burden and potentially increase T-cell re-invigoration (50,51). Moreover, increased volume and diversity of the tumor antigens released following multi-site SBRT makes this approach potentially appealing. On the whole, these theoretical advantages of multi-site SBRT along with the fact that immunotherapy has been demonstrated to be most efficacious in the setting of lowvolume disease (49) support the potential utility of treating several metastatic sites in an attempt to improve the efficacy of SBRT combined with ICB.

In summary, recent advancements in the understanding, planning, and delivery of metastasis-directed radiation therapy have increased the feasibility of treating multiple metastatic sites with ablative doses of radiation. Continued innovations in treatment planning and delivery, such as autonomous contouring (145) and biology-guided radiation therapy (146) have the potential to continue increase the feasibility of treating multiple metastases with ablative doses. Moreover, given data that the decreased absolute lymphocyte count (ALC) at the completion of SBRT is associated decreased rates of response to ICB (147), treatment planning approaches which limit "low dose bath" to surrounding tissues may be employed in order to decrease post-treatment lymphopenia (148). Future studies 
utilizing these techniques are needed in order to further investigate how to optimize rates of response to combined SBRT and ICB.

\section{Defining the population likely to benefit from SBRT and ICB}

Another potential strategy to improve the therapeutic ratio of combined SBRT and ICB in patients with oligometastatic disease is to identify the subpopulation of patients most likely to benefit from this approach. Clinical factors associated with a favorable long-term prognosis among patients with oligometastatic disease treated with metastasis-directed therapy are well-established $(11,12)$. More recently, molecular factors associated with oligoversus polymetastatic progression have been identified (149) providing the potential to discern which patients are likely to be cured by metastasis-directed therapy, versus those who are likely to fail diffusely. Notably, Pitroda et al. recently proposed an integrated molecular classification in patients with hepatic metastases from colorectal cancer that successfully stratified patients by risk of failure following metastasectomy (150). Not only does this study provide a potentially useful framework upon which patients with curable oligometastatic disease could be successfully identified, but given that patients in whom microsatellite instability-independent immune activation was exhibited had the most favorable long-term outcome in this analysis, this framework has potentially important implications for the use of immunotherapy in patients with oligometastatic disease. Overall, efforts to further define the population most likely to benefit from the combination of SBRT and ICB are needed for this therapeutic approach to be utilized in a manner that maximizes its benefit to risk ratio.

\section{Conclusions}

Patients with oligometastatic cancer represent a subset of the metastatic population with a limited amount of disease that has metastasized distantly but is unlikely to progress rapidly and thus has the potential to be cured with metastasis-directed local therapies, such as SBRT. While initial evidence suggests that SBRT may provide significant benefit in patients with oligometastatic disease, high rates of distant failure following local therapy continue to preclude long-term disease control in this population as a whole. ICB has proven benefits in many metastatic cancers and may be beneficial in combination with SBRT in the oligometastatic population. Based upon short-term follow-up from prospective studies, the combination of SBRT with ICB appears to be safe with preliminary, hypothesis-generating data suggesting potential efficacy; however, longer followup and additional studies are needed investigate this further. Going forward, prospective, randomized studies evaluating the utility of multi-site, metastasis-directed SBRT utilizing ablative doses in combination with ICB are needed to better evaluate the efficacy of this approach and how the therapeutic ratio of combined-modality therapy can be maximized.

\section{Acknowledgments}

Funding: None.

\section{Footnote}

Provenance and Peer Review: This article was commissioned by the Guest Editors (Simon Lo, Michael Milano, Tithi Biswas, Charles Simone) for the series "OligometastasisFallacy or Real Deal?" published in Annals of Palliative Medicine. The article has undergone external peer review.

Reporting Checklist: The authors have completed the NARRATIVE REVIEW reporting checklist. Available at http://dx.doi.org/10.21037/apm-20-1528

Conflicts of Interest: Both authors have completed the ICMJE uniform disclosure form (available at http://dx.doi. org/10.21037/apm-20-1528). The series "OligometastasisFallacy or Real Deal?" was commissioned by the editorial office without any funding or sponsorship. SJC reports personal fees from RefleXion Medical, other from Astellas Pharma, and grants from Merck and BMS outside the submitted work. The authors have no other conflicts of interest to declare.

Ethical Statement: The authors are accountable for all aspects of the work in ensuring that questions related to the accuracy or integrity of any part of the work are appropriately investigated and resolved.

Open Access Statement: This is an Open Access article distributed in accordance with the Creative Commons Attribution-NonCommercial-NoDerivs 4.0 International License (CC BY-NC-ND 4.0), which permits the noncommercial replication and distribution of the article with 
the strict proviso that no changes or edits are made and the original work is properly cited (including links to both the formal publication through the relevant DOI and the license). See: https://creativecommons.org/licenses/by-nc-nd/4.0/.

\section{References}

1. Seyfried TN, Huysentruyt LC. On the origin of cancer metastasis. Crit Rev Oncog 2013;18:43-73.

2. Halsted WS. I. The Results of Radical Operations for the Cure of Carcinoma of the Breast. Ann Surg 1907;46:1-19.

3. Fisher B. Laboratory and clinical research in breast cancer--a personal adventure: the David A. Karnofsky memorial lecture. Cancer Res 1980;40:3863-74.

4. Hellman S. Karnofsky Memorial Lecture. Natural history of small breast cancers. J Clin Oncol 1994;12:2229-34.

5. Hellman S, Weichselbaum RR. Oligometastases. J Clin Oncol 1995;13:8-10.

6. Dawood S, Broglio K, Gonzalez-Angulo AM, et al. Trends in survival over the past two decades among white and black patients with newly diagnosed stage IV breast cancer. J Clin Oncol 2008;26:4891-8.

7. Mehta N, Mauer AM, Hellman S, et al. Analysis of further disease progression in metastatic non-small cell lung cancer: implications for locoregional treatment. Int J Oncol 2004;25:1677-83.

8. Tosoian JJ, Gorin MA, Ross AE, et al. Oligometastatic prostate cancer: definitions, clinical outcomes, and treatment considerations. Nat Rev Urol 2017;14:15-25.

9. Loh J, Davis ID, Martin JM, et al. Extracranial oligometastatic renal cell carcinoma: current management and future directions. Future Oncol 2014;10:761-74.

10. Weinlechner J. Tumoren an der brustwand und deren behnadlung resection der rippeneroffnung der brusthohle und partielle entfernung der lunge. Wien Med Wochenschr 1882;32:589-91.

11. Fong Y, Fortner J, Sun RL, et al. Clinical score for predicting recurrence after hepatic resection for metastatic colorectal cancer: analysis of 1001 consecutive cases. Ann Surg 1999;230:309-18; discussion 318-21.

12. Tomlinson JS, Jarnagin WR, DeMatteo RP, et al. Actual 10-year survival after resection of colorectal liver metastases defines cure. J Clin Oncol 2007;25:4575-80.

13. Pastorino U, Buyse M, Friedel G, et al. Long-term results of lung metastasectomy: prognostic analyses based on 5206 cases. J Thorac Cardiovasc Surg 1997;113:37-49.

14. Weichselbaum RR, Hellman S. Oligometastases revisited. Nat Rev Clin Oncol 2011;8:378-82.
15. Lewis SL, Porceddu S, Nakamura N, et al. Definitive Stereotactic Body Radiotherapy (SBRT) for Extracranial Oligometastases: An International Survey of $>1000$ Radiation Oncologists. Am J Clin Oncol 2017;40:418-22.

16. Milano MT, Katz AW, Zhang H, et al. Oligometastatic breast cancer treated with hypofractionated stereotactic radiotherapy: Some patients survive longer than a decade. Radiother Oncol 2019;131:45-51.

17. Milano MT, Katz AW, Zhang H, et al. Oligometastases treated with stereotactic body radiotherapy: long-term follow-up of prospective study. Int J Radiat Oncol Biol Phys 2012;83:878-86.

18. Rusthoven KE, Kavanagh BD, Burri SH, et al. Multiinstitutional phase I/II trial of stereotactic body radiation therapy for lung metastases. J Clin Oncol 2009;27:1579-84.

19. Rusthoven KE, Kavanagh BD, Cardenes H, et al. Multiinstitutional phase I/II trial of stereotactic body radiation therapy for liver metastases. J Clin Oncol 2009;27:1572-8.

20. Salama JK, Hasselle MD, Chmura SJ, et al. Stereotactic body radiotherapy for multisite extracranial oligometastases: final report of a dose escalation trial in patients with 1 to 5 sites of metastatic disease. Cancer 2012;118:2962-70.

21. Palma DA, Olson R, Harrow S, et al. Stereotactic ablative radiotherapy versus standard of care palliative treatment in patients with oligometastatic cancers (SABRCOMET): a randomised, phase 2, open-label trial. Lancet 2019;393:2051-8.

22. Palma DA, Olson R, Harrow S, et al. Stereotactic Ablative Radiotherapy for the Comprehensive Treatment of Oligometastatic Cancers: Long-Term Results of the SABR-COMET Phase II Randomized Trial. J Clin Oncol 2020;38:2830-8.

23. Rubinstein LV, Korn EL, Freidlin B, et al. Design issues of randomized phase II trials and a proposal for phase II screening trials. J Clin Oncol 2005;23:7199-206.

24. Gomez DR, Tang C, Zhang J, et al. Local Consolidative Therapy Vs. Maintenance Therapy or Observation for Patients With Oligometastatic Non-Small-Cell Lung Cancer: Long-Term Results of a Multi-Institutional, Phase II, Randomized Study. J Clin Oncol 2019;37:1558-65.

25. Iyengar $P$, Wardak Z, Gerber DE, et al. Consolidative Radiotherapy for Limited Metastatic Non-Small-Cell Lung Cancer: A Phase 2 Randomized Clinical Trial. JAMA Oncol 2018;4:e173501.

26. Ost P, Reynders D, Decaestecker K, et al. Surveillance or Metastasis-Directed Therapy for Oligometastatic Prostate Cancer Recurrence: A Prospective, Randomized, 
Multicenter Phase II Trial. J Clin Oncol 2018;36:446-53.

27. Phillips R, Shi WY, Deek M, et al. Outcomes of Observation vs Stereotactic Ablative Radiation for Oligometastatic Prostate Cancer: The ORIOLE Phase 2 Randomized Clinical Trial. JAMA Oncol 2020;6:650-9.

28. Cardoso F, Costa A, Senkus E, et al. 3rd ESO-ESMO International Consensus Guidelines for Advanced Breast Cancer (ABC 3). Ann Oncol 2017;28:16-33.

29. Cameron F, Whiteside G, Perry C. Ipilimumab: first global approval. Drugs 2011;71:1093-104.

30. Haslam A, Prasad V. Estimation of the Percentage of US Patients With Cancer Who Are Eligible for and Respond to Checkpoint Inhibitor Immunotherapy Drugs. JAMA Netw Open 2019;2:e192535.

31. Gandhi L, Rodriguez-Abreu D, Gadgeel S, et al. Pembrolizumab plus Chemotherapy in Metastatic NonSmall-Cell Lung Cancer. N Engl J Med 2018;378:2078-92.

32. Garon EB, Rizvi NA, Hui R, et al. Pembrolizumab for the treatment of non-small-cell lung cancer. N Engl J Med 2015;372:2018-28.

33. Paz-Ares L, Luft A, Vicente D, et al. Pembrolizumab plus Chemotherapy for Squamous Non-Small-Cell Lung Cancer. N Engl J Med 2018;379:2040-51.

34. Reck M, Brahmer JR. Pembrolizumab in Non-Small-Cell Lung Cancer. N Engl J Med 2017;376:997.

35. Reck M, Rodriguez-Abreu D, Robinson AG, et al. Pembrolizumab versus Chemotherapy for PD-L1Positive Non-Small-Cell Lung Cancer. N Engl J Med 2016;375:1823-33.

36. Hamid O, Robert C, Daud A, et al. Five-year survival outcomes for patients with advanced melanoma treated with pembrolizumab in KEYNOTE-001. Ann Oncol 2019;30:582-8.

37. Motzer RJ, Tannir NM, McDermott DF, et al. Nivolumab plus Ipilimumab versus Sunitinib in Advanced Renal-Cell Carcinoma. N Engl J Med 2018;378:1277-90.

38. Horn L, Mansfield AS, Szczesna A, et al. First-Line Atezolizumab plus Chemotherapy in Extensive-Stage Small-Cell Lung Cancer. N Engl J Med 2018;379:2220-9.

39. Brahmer J, Reckamp KL, Baas P, et al. Nivolumab versus Docetaxel in Advanced Squamous-Cell Non-Small-Cell Lung Cancer. N Engl J Med 2015;373:123-35.

40. Motzer RJ, Escudier B, McDermott DF, et al. Nivolumab versus Everolimus in Advanced Renal-Cell Carcinoma. N Engl J Med 2015;373:1803-13.

41. Motzer RJ, Rini BI, McDermott DF, et al. Nivolumab for Metastatic Renal Cell Carcinoma: Results of a Randomized Phase II Trial. J Clin Oncol 2015;33:1430-7.
42. Rosenberg JE, Hoffman-Censits J, Powles T, et al. Atezolizumab in patients with locally advanced and metastatic urothelial carcinoma who have progressed following treatment with platinum-based chemotherapy: a single-arm, multicentre, phase 2 trial. Lancet 2016;387:1909-20.

43. Chen DS, Mellman I. Elements of cancer immunity and the cancer-immune set point. Nature 2017;541:321-30.

44. Khoja L, Butler MO, Kang SP, et al. Pembrolizumab. J Immunother Cancer 2015;3:36.

45. Schulze AB, Schmidt LH. PD-1 targeted Immunotherapy as first-line therapy for advanced non-small-cell lung cancer patients. J Thorac Dis 2017;9:E384-6.

46. Zaretsky JM, Garcia-Diaz A, Shin DS, et al. Mutations Associated with Acquired Resistance to PD-1 Blockade in Melanoma. N Engl J Med 2016;375:819-29.

47. Antonia SJ, Villegas A, Daniel D, et al. Overall Survival with Durvalumab after Chemoradiotherapy in Stage III NSCLC. N Engl J Med 2018;379:2342-50.

48. Antonia SJ, Villegas A, Daniel D, et al. Durvalumab after Chemoradiotherapy in Stage III Non-Small-Cell Lung Cancer. N Engl J Med 2017;377:1919-29.

49. Predina JD, Judy B, Aliperti LA, et al. Neoadjuvant in situ gene-mediated cytotoxic immunotherapy improves postoperative outcomes in novel syngeneic esophageal carcinoma models. Cancer Gene Ther 2011;18:871-83.

50. Wherry EJ, Blattman JN, Murali-Krishna K, et al. Viral persistence alters CD8 T-cell immunodominance and tissue distribution and results in distinct stages of functional impairment. J Virol 2003;77:4911-27.

51. Blackburn SD, Shin H, Haining WN, et al. Coregulation of CD8+ T cell exhaustion by multiple inhibitory receptors during chronic viral infection. Nat Immunol 2009;10:29-37.

52. Mole RH. Whole body irradiation; radiobiology or medicine? Br J Radiol 1953;26:234-41.

53. Reynders K, Illidge T, Siva S, et al. The abscopal effect of local radiotherapy: using immunotherapy to make a rare event clinically relevant. Cancer Treat Rev 2015;41:503-10.

54. Demaria $\mathrm{S}, \mathrm{Ng}$ B, Devitt $\mathrm{ML}$, et al. Ionizing radiation inhibition of distant untreated tumors (abscopal effect) is immune mediated. Int J Radiat Oncol Biol Phys 2004;58:862-70.

55. Postow MA, Callahan MK, Barker CA, et al. Immunologic correlates of the abscopal effect in a patient with melanoma. N Engl J Med 2012;366:925-31.

56. Jiménez-Sánchez A, Memon D, Pourpe S, et al. Heterogeneous Tumor-Immune Microenvironments 
among Differentially Growing Metastases in an Ovarian Cancer Patient. Cell 2017;170:927-38.e20.

57. Lee Y, Auh SL, Wang Y, Burnette B, Wang Y, Meng Y, et al. Therapeutic effects of ablative radiation on local tumor require $\mathrm{CD} 8+\mathrm{T}$ cells: changing strategies for cancer treatment. Blood 2009;114:589-95.

58. Demaria S, Formenti SC. Role of T lymphocytes in tumor response to radiotherapy. Front Oncol 2012;2:95.

59. Gupta A, Probst HC, Vuong V, et al. Radiotherapy promotes tumor-specific effector CD8+ T cells via dendritic cell activation. J Immunol 2012;189:558-66.

60. Anitei MG, Zeitoun G, Mlecnik B, et al. Prognostic and predictive values of the immunoscore in patients with rectal cancer. Clin Cancer Res 2014;20:1891-9.

61. Characiejus D, Jacobs JJ, Pasukoniene V, et al. Prediction of response in cancer immunotherapy. Anticancer Res 2011;31:639-47.

62. Characiejus D, Pasukoniene V, Jacobs JJ, et al. Prognostic significance of peripheral blood CD8highCD57+ lymphocytes in bladder carcinoma patients after intravesical IL-2. Anticancer Res 2011;31:699-703.

63. Liu S, Lachapelle J, Leung S, et al. CD8+ lymphocyte infiltration is an independent favorable prognostic indicator in basal-like breast cancer. Breast Cancer Res 2012;14:R48.

64. Tumeh PC, Harview CL, Yearley JH, et al. PD-1 blockade induces responses by inhibiting adaptive immune resistance. Nature 2014;515:568-71.

65. Nishino M, Ramaiya NH, Hatabu H, et al. Monitoring immune-checkpoint blockade: response evaluation and biomarker development. Nat Rev Clin Oncol 2017;14:655-68.

66. Syn NL, Teng MWL, Mok TSK, et al. De-novo and acquired resistance to immune checkpoint targeting. Lancet Oncol 2017;18:e731-41.

67. Deng L, Liang H, Fu S, et al. From DNA Damage to Nucleic Acid Sensing: A Strategy to Enhance Radiation Therapy. Clin Cancer Res 2016;22:20-5.

68. Sun L, Wu J, Du F, et al. Cyclic GMP-AMP synthase is a cytosolic DNA sensor that activates the type I interferon pathway. Science 2013;339:786-91.

69. Weichselbaum RR, Ishwaran H, Yoon T, et al. An interferon-related gene signature for DNA damage resistance is a predictive marker for chemotherapy and radiation for breast cancer. Proc Natl Acad Sci U S A 2008;105:18490-5.

70. Deng L, Liang H, Burnette B, et al. Irradiation and antiPD-L1 treatment synergistically promote antitumor immunity in mice. J Clin Invest 2014;124:687-95.

71. Chen MF, Chen PT, Chen WC, et al. The role of PD$\mathrm{L} 1$ in the radiation response and prognosis for esophageal squamous cell carcinoma related to IL-6 and T-cell immunosuppression. Oncotarget 2016;7:7913-24.

72. Dovedi SJ, Adlard AL, Lipowska-Bhalla G, et al. Acquired resistance to fractionated radiotherapy can be overcome by concurrent PD-L1 blockade. Cancer Res 2014;74:5458-68.

73. Lugade AA, Moran JP, Gerber SA, et al. Local radiation therapy of $\mathrm{B} 16$ melanoma tumors increases the generation of tumor antigen-specific effector cells that traffic to the tumor. J Immunol 2005;174:7516-23.

74. Matsumura S, Wang B, Kawashima N, et al. Radiationinduced CXCL16 release by breast cancer cells attracts effector T cells. J Immunol 2008;181:3099-107.

75. Twyman-Saint Victor C, Rech AJ, Maity A, et al. Radiation and dual checkpoint blockade activate non-redundant immune mechanisms in cancer. Nature 2015;520:373-7.

76. Klug F, Prakash H, Huber PE, et al. Low-dose irradiation programs macrophage differentiation to an iNOS(+)/ M1 phenotype that orchestrates effective $T$ cell immunotherapy. Cancer Cell 2013;24:589-602.

77. Liang H, Deng L, Hou Y, et al. Host STING-dependent MDSC mobilization drives extrinsic radiation resistance. Nat Commun 2017;8:1736.

78. Deng L, Liang H, Xu M, et al. STING-Dependent Cytosolic DNA Sensing Promotes Radiation-Induced Type I Interferon-Dependent Antitumor Immunity in Immunogenic Tumors. Immunity 2014;41:843-52.

79. Arina A, Beckett M, Fernandez C, et al. Tumorreprogrammed resident $T$ cells resist radiation to control tumors. Nat Commun 2019;10:3959.

80. Schaue D, Kachikwu EL, McBride WH. Cytokines in radiobiological responses: a review. Radiat Res 2012;178:505-23.

81. Kachikwu EL, Iwamoto KS, Liao YP, et al. Radiation enhances regulatory $\mathrm{T}$ cell representation. Int J Radiat Oncol Biol Phys 2011;81:1128-35.

82. Persa E, Balogh A, Safrany G, et al. The effect of ionizing radiation on regulatory $T$ cells in health and disease. Cancer Lett 2015;368:252-61.

83. Ahn GO, Tseng D, Liao CH, et al. Inhibition of Mac-1 (CD11b/CD18) enhances tumor response to radiation by reducing myeloid cell recruitment. Proc Natl Acad Sci U S A 2010;107:8363-8.

84. Burnette BC, Liang H, Lee Y, et al. The efficacy of radiotherapy relies upon induction of type $\mathrm{i}$ interferon- 
dependent innate and adaptive immunity. Cancer Res 2011;71:2488-96.

85. Burnette B, Weichselbaum RR. Radiation as an immune modulator. Semin Radiat Oncol 2013;23:273-80.

86. Facciabene A, Motz GT, Coukos G. T-regulatory cells: key players in tumor immune escape and angiogenesis. Cancer Res 2012;72:2162-71.

87. Qinfeng S, Depu W, Xiaofeng Y, et al. In situ observation of the effects of local irradiation on cytotoxic and regulatory $\mathrm{T}$ lymphocytes in cervical cancer tissue. Radiat Res 2013;179:584-9.

88. Schuler PJ, Harasymczuk M, Schilling B, et al. Effects of adjuvant chemoradiotherapy on the frequency and function of regulatory $T$ cells in patients with head and neck cancer. Clin Cancer Res 2013;19:6585-96.

89. Condamine T, Ramachandran I, Youn JI, et al. Regulation of tumor metastasis by myeloid-derived suppressor cells. Annu Rev Med 2015;66:97-110.

90. Lindau D, Gielen P, Kroesen M, et al. The immunosuppressive tumour network: myeloid-derived suppressor cells, regulatory $\mathrm{T}$ cells and natural killer $\mathrm{T}$ cells. Immunology 2013;138:105-15.

91. Tang F, Du X, Liu M, et al. Anti-CTLA-4 antibodies in cancer immunotherapy: selective depletion of intratumoral regulatory $\mathrm{T}$ cells or checkpoint blockade? Cell Biosci 2018;8:30.

92. Mills CD. M1 and M2 Macrophages: Oracles of Health and Disease. Crit Rev Immunol 2012;32:463-88.

93. Martinez FO, Gordon S. The M1 and M2 paradigm of macrophage activation: time for reassessment. F1000Prime Rep 2014;6:13.

94. Seifert L, Werba G, Tiwari S, et al. Radiation Therapy Induces Macrophages to Suppress T-Cell Responses Against Pancreatic Tumors in Mice. Gastroenterology 2016;150:1659-72.e5.

95. Chiang CS, Fu SY, Wang SC, et al. Irradiation promotes an $\mathrm{m} 2$ macrophage phenotype in tumor hypoxia. Front Oncol 2012;2:89.

96. Meng Y, Beckett MA, Liang H, et al. Blockade of tumor necrosis factor alpha signaling in tumor-associated macrophages as a radiosensitizing strategy. Cancer Res 2010;70:1534-43.

97. Zhu Y, Knolhoff BL, Meyer MA, et al. CSF1/CSF1R blockade reprograms tumor-infiltrating macrophages and improves response to T-cell checkpoint immunotherapy in pancreatic cancer models. Cancer Res 2014;74:5057-69.

98. Obeid M, Tesniere A, Ghiringhelli F, et al. Calreticulin exposure dictates the immunogenicity of cancer cell death.
Nat Med 2007;13:54-61.

99. Krysko DV, Garg AD, Kaczmarek A, et al. Immunogenic cell death and DAMPs in cancer therapy. Nat Rev Cancer 2012;12:860-75.

100.Huang AC, Postow MA, Orlowski RJ, et al. T-cell invigoration to tumour burden ratio associated with antiPD-1 response. Nature 2017;545:60-5.

101. Naidoo J, Page DB, Li BT, et al. Toxicities of the antiPD-1 and anti-PD-L1 immune checkpoint antibodies. Ann Oncol 2015;26:2375-91.

102. Weber JS, Dummer R, de Pril V, et al. Patterns of onset and resolution of immune-related adverse events of special interest with ipilimumab: detailed safety analysis from a phase 3 trial in patients with advanced melanoma. Cancer 2013;119:1675-82.

103. Barroso-Sousa R, Barry WT, Garrido-Castro AC, et al. Incidence of Endocrine Dysfunction Following the Use of Different Immune Checkpoint Inhibitor Regimens: A Systematic Review and Meta-analysis. JAMA Oncol 2018;4:173-82.

104. Tsung I, Dolan R, Lao CD, et al. Liver injury is most commonly due to hepatic metastases rather than drug hepatotoxicity during pembrolizumab immunotherapy. Aliment Pharmacol Ther 2019;50:800-8.

105. Nishino M, Giobbie-Hurder A, Hatabu H, et al. Incidence of Programmed Cell Death 1 InhibitorRelated Pneumonitis in Patients With Advanced Cancer: A Systematic Review and Meta-analysis. JAMA Oncol 2016;2:1607-16.

106. Nishino M, Ramaiya NH, Awad MM, et al. PD-1 Inhibitor-Related Pneumonitis in Advanced Cancer Patients: Radiographic Patterns and Clinical Course. Clin Cancer Res 2016;22:6051-60.

107. Naidoo J, Wang X, Woo KM, et al. Pneumonitis in Patients Treated With Anti-Programmed Death-1/ Programmed Death Ligand 1 Therapy. J Clin Oncol 2017;35:709-17.

108. Delaunay M, Cadranel J, Lusque A, et al. Immunecheckpoint inhibitors associated with interstitial lung disease in cancer patients. Eur Respir J 2017;50:1700050.

109. Khunger M, Rakshit S, Pasupuleti V, et al. Incidence of Pneumonitis With Use of Programmed Death 1 and Programmed Death-Ligand 1 Inhibitors in Non-Small Cell Lung Cancer: A Systematic Review and MetaAnalysis of Trials. Chest 2017;152:271-81.

110. Shibaki R, Akamatsu H, Fujimoto M, et al. Nivolumab induced radiation recall pneumonitis after two years of radiotherapy. Ann Oncol 2017;28:1404-5. 
111.Luke JJ, Lemons JM, Karrison TG, et al. Safety and Clinical Activity of Pembrolizumab and Multisite Stereotactic Body Radiotherapy in Patients With Advanced Solid Tumors. J Clin Oncol 2018;36:1611-8.

112. Onderdonk BE, Luke JJ, Bhave SR, et al. Multi-Site SBRT and Sequential Pembrolizumab: Treated Metastasis Control and Immune-Related Expression Predict Outcomes. Int J Radiat Oncol Biol Phys 2019;104:1190-1.

113. Tang C, Welsh JW, de Groot P, et al. Ipilimumab with Stereotactic Ablative Radiation Therapy: Phase I Results and Immunologic Correlates from Peripheral T Cells. Clin Cancer Res 2017;23:1388-96.

114. Williams NL, Wuthrick EJ, Kim H, et al. Phase 1 Study of Ipilimumab Combined With Whole Brain Radiation Therapy or Radiosurgery for Melanoma Patients With Brain Metastases. Int J Radiat Oncol Biol Phys 2017;99:22-30.

115. Slovin SF, Higano CS, Hamid O, et al. Ipilimumab alone or in combination with radiotherapy in metastatic castration-resistant prostate cancer: results from an open-label, multicenter phase I/II study. Ann Oncol 2013;24:1813-21.

116. Kwon ED, Drake CG, Scher HI, et al. Ipilimumab versus placebo after radiotherapy in patients with metastatic castration-resistant prostate cancer that had progressed after docetaxel chemotherapy (CA184-043): a multicentre, randomised, double-blind, phase 3 trial. Lancet Oncol 2014;15:700-12.

117.Hiniker SM, Reddy SA, Maecker HT, et al. A Prospective Clinical Trial Combining Radiation Therapy With Systemic Immunotherapy in Metastatic Melanoma. Int J Radiat Oncol Biol Phys 2016;96:578-88.

118. Campbell AM, Herbst RS, Gettinger SN, et al. Final results of a phase I prospective trial evaluating the combination of stereotactic body radiotherapy (SBRT) with concurrent pembrolizumab in patients with metastatic non-small cell lung cancer (NSCLC) or melanoma. J Clin Oncol 2018;36:9099.

119. Robinson C, Hu C, Machtay M, et al. P1.18-12 PACIFIC-4/RTOG 3515: Phase III Study of Durvalumab Following SBRT for Unresected Stage I/II, Lymph-Node Negative NSCLC. J Thorac Oncol 2019;14:S630-1.

120. Kelly K, Daly ME, Mirhadi A, et al. Atezolizumab plus stereotactic ablative therapy for medically inoperable patients with early-stage non-small cell lung cancer. J Clin Oncol 2020;38:9011.

121. Theelen WS, Peulen HMU, Lalezari F, et al. Effect of Pembrolizumab After Stereotactic Body Radiotherapy vs
Pembrolizumab Alone on Tumor Response in Patients With Advanced Non-Small Cell Lung Cancer: Results of the PEMBRO-RT Phase 2 Randomized Clinical Trial. JAMA Oncol 2019;5:1276-82.

122. Welsh J, Menon H, Chen D, et al. Pembrolizumab with or without radiation therapy for metastatic non-small cell lung cancer: a randomized phase I/II trial. J Immunother Cancer 2020;8:e001001.

123. Theelen WSME, Chen D, Verma V, et al. Pembrolizumab with or without radiotherapy for metastatic non-small-cell lung cancer: a pooled analysis of two randomised trials. Lancet Respir Med 2020;S2213-2600(20)30391-X.

124.McBride S, Sherman E, Tsai CJ, et al. Randomized Phase II Trial of Nivolumab With Stereotactic Body Radiotherapy Versus Nivolumab Alone in Metastatic Head and Neck Squamous Cell Carcinoma. J Clin Oncol 2021;39:30-7.

125.Moreno V, Gil-Martin M, Johnson M, et al. MA04.01 Cemiplimab, a Human Monoclonal Anti-PD-1, Alone or in Combination with Radiotherapy: Phase 1 NSCLC Expansion Cohorts. J Thorac Oncol 2018;13:S366.

126.Park S, Urm S, Cho H. Analysis of biologically equivalent dose of stereotactic body radiotherapy for primary and metastatic lung tumors. Cancer Res Treat 2014;46:403-10.

127. Ricco A, Davis J, Rate W, et al. Lung metastases treated with stereotactic body radiotherapy: the RSSearch(R) patient Registry's experience. Radiat Oncol 2017;12:35.

128. Mahadevan A, Blanck O, Lanciano R, et al. Stereotactic Body Radiotherapy (SBRT) for liver metastasis - clinical outcomes from the international multi-institutional RSSearch(R) Patient Registry. Radiat Oncol 2018;13:26.

129.Zhao X, Zhu X, Zhuang H, et al. Clinical efficacy of Stereotactic Body Radiation Therapy (SBRT) for adrenal gland metastases: A multi-center retrospective study from China. Sci Rep 2020;10:7836.

130. Golden EB, Chhabra A, Chachoua A, et al. Local radiotherapy and granulocyte-macrophage colonystimulating factor to generate abscopal responses in patients with metastatic solid tumours: a proof-of-principle trial. Lancet Oncol 2015;16:795-803.

131. Wainberg Z, Piha-Paul S, Luke J, et al. First-in-Human Phase 1 Dose Escalation and Expansion of a Novel Combination, Anti-CSF-1 Receptor (cabiralizumab) Plus Anti-PD-1 (nivolumab), in Patients With Advanced Solid Tumors. 2018.

132. Shi W, Siemann DW. Augmented antitumor effects of radiation therapy by 4-1BB antibody (BMS-469492) treatment. Anticancer Res 2006;26:3445-53. 
133. Kroon P, Gadiot J, Peeters M, et al. Concomitant targeting of programmed death-1 (PD-1) and CD137 improves the efficacy of radiotherapy in a mouse model of human BRAFV600-mutant melanoma. Cancer Immunol Immunother 2016;65:753-63.

134. Shiao JC, Bowers N, Nasti TH, et al. 4-1BB (CD137) and radiation therapy: A case report and literature review. Adv Radiat Oncol 2017;2:398-402.

135. Tolcher AW, Sznol M, Hu-Lieskovan S, et al. Phase Ib Study of Utomilumab (PF-05082566), a 4-1BB/CD137 Agonist, in Combination with Pembrolizumab (MK-3475) in Patients with Advanced Solid Tumors. Clin Cancer Res 2017;23:5349-57.

136. Mariathasan S, Turley SJ, Nickles D, et al. TGFbeta attenuates tumour response to PD-L1 blockade by contributing to exclusion of $\mathrm{T}$ cells. Nature 2018;554:544-8.

137. Grenga I, Donahue RN, Gargulak ML, et al. Anti-PD-L1/ TGFbetaR2 (M7824) fusion protein induces immunogenic modulation of human urothelial carcinoma cell lines, rendering them more susceptible to immune-mediated recognition and lysis. Urol Oncol 2018;36:93.e1-e11.

138. David JM, Dominguez C, McCampbell KK, et al. A novel bifunctional anti-PD-L1/TGF-beta Trap fusion protein (M7824) efficiently reverts mesenchymalization of human lung cancer cells. Oncoimmunology 2017;6:e1349589.

139. Shergold AL, Millar R, Nibbs RJB. Understanding and overcoming the resistance of cancer to PD-1/PD-L1 blockade. Pharmacol Res 2019;145:104258.

140. Chmura SJ, Winter K, Salama JK, et al. Phase I Trial of Stereotactic Body Radiation Therapy (SBRT) to Multiple Metastatic Sites: A NRG Oncology Study. Int J Radiat Oncol Biol Phys 2018;102:S68-9.

141. Teoh M, Clark CH, Wood K, et al. Volumetric modulated arc therapy: a review of current literature and clinical use in practice. Br J Radiol 2011;84:967-96.

142. Pointer KB, Rokni M, Luke JJ, et al. Feasibility of

Cite this article as: Turchan WT, Chmura SJ. The role of immunotherapy in combination with oligometastasis-directed therapy: a narrative review. Ann Palliat Med 2021;10(5):6028-6044. doi: 10.21037/apm-20-1528
Delivering Immunotherapy with Concomittant Ablative Radiosurgery to Ultra-high DoSes (ICARUS). Int J Radiat Oncol Biol Phys 2019;105:Supplement:E771.

143.McBride SM, Sherman EJ, Tsai CJ, et al. A phase II randomized trial of nivolumab with stereotactic body radiotherapy (SBRT) versus nivolumab alone in metastatic (M1) head and neck squamous cell carcinoma (HNSCC). J Clin Oncol 2018;36:6009.

144. Wray J, Hawamdeh RF, Hasija N, et al. Stereotactic body radiation therapy for oligoprogression of metastatic disease from gastrointestinal cancers: A novel approach to extend chemotherapy efficacy. Oncol Lett 2017;13:1087-94.

145.Hussein M, Heijmen BJM, Verellen D, et al. Automation in intensity modulated radiotherapy treatment planning-a review of recent innovations. Br J Radiol 2018;91:20180270.

146.Hrinivich WT, Phillips R, Da Silva AJ, et al. Online Prostate-Specific Membrane Antigen and Positron Emission Tomography-Guided Radiation Therapy for Oligometastatic Prostate Cancer. Adv Radiat Oncol 2019;5:260-8.

147. Chen D, Verma V, Patel RR, et al. Absolute Lymphocyte Count Predicts Abscopal Responses and Outcomes in Patients Receiving Combined Immunotherapy and Radiation Therapy: Analysis of 3 Phase 1/2 Trials. Int J Radiat Oncol Biol Phys 2020;108:196-203.

148. Tang C, Liao Z, Gomez D, et al. Lymphopenia Association With Gross Tumor Volume and Lung V5 and Its Effects on Non-Small Cell Lung Cancer Patient Outcomes. Int J Radiat Oncol Biol Phys 2014;89:1084-91.

149.Lussier YA, Khodarev NN, Regan K, et al. Oligo- and polymetastatic progression in lung metastasis(es) patients is associated with specific microRNAs. PLoS One 2012;7:e50141.

150.Pitroda SP, Khodarev NN, Huang L, et al. Integrated molecular subtyping defines a curable oligometastatic state in colorectal liver metastasis. Nat Commun 2018;9:1793. 